Produto \& Produção, vol. 13 n. 1, p. 16-33, fev. 2012

\title{
Métodos e ferramentas de Ecodesign: revisão bibliográfica sistemática
}

\author{
Recebido em 30/10/2011. Aceito em 05/01/2012.
}

\section{Daniela Pigosso}

Escola de Engenharia de São Carlos - USP

daniela.pigosso@gmail.com

\section{Henrique Rozenfeld \\ Escola de Engenharia de São Carlos - USP \\ roz@sc.usp.br}

Todos os produtos causam impactos ambientais ao longo do seu ciclo de vida, da extração da matéria-prima até a sua disposição final. O ecodesign, uma abordagem pró-ativa de gestão ambiental, atua no desenvolvimento de produtos de forma a minimizar os impactos ambientais durante todo o ciclo de vida dos produtos. A não obtenção das potencialidades do ecodesign, como previsto na década de 1990, pode ser explicada pelo intenso desenvolvimento de novos métodos e ferramentas de ecodesign em detrimento do estudo e aprimoramento das existentes. Este trabalho teve como objetivo o levantamento do estado da arte, a classificação e a sistematização dos métodos e ferramentas de ecodesign por meio da realização de uma revisão bibliográfica sistemática. A classificação realizada pode contribuir com a seleção dos métodos mais adequados para cada empresa, de acordo com o seu direcionamento estratégico e com os produtos que são desenvolvidos.

Palavras chave: Ecodesign; processo de desenvolvimento de produto; métodos e ferramentas.

Products cause environmental impacts throughout its life cycle, from raw material extraction to end-of-life disposal. Ecodesign, a proactive approach to environmental management, acts in product development by minimizing environmental impacts throughout the product's life cycle. The non-achievement of ecodesign potentialities as foreseen in the 1990s may be explained by the intense development of new ecodesign methods and tools in detriment to the study and improvement of existing ones. The purpose of this work was to make a survey of the state of the art, and a classification and systematization of ecodesign methods and tools based on a systematic review of the literature. The classification drawn up here may contribute to the selection of the most adequate methods by companies, according to their strategic orientation and the products they develop.

Keywords: ecodesign; product development process; methods and tools. 


\section{INTRODUÇÃO E JUSTIFICATIVA}

Os produtos são fundamentais para a riqueza da sociedade e para a qualidade de vida desejada. Todavia, o crescente consumo de produtos está também, direta ou indiretamente, na origem da maior parte da poluição e do esgotamento de recursos que a sociedade causa (COMISSION OF THE EUROPEAN COMMUNNITIES, 2001).

O ecodesign, uma abordagem de gestão ambiental pró-ativa, corresponde à consideração de questões ambientais no processo de desenvolvimento de produtos direcionadas à minimização dos impactos ambientais durante todo o ciclo de vida do produto, sem comprometer outros critérios essenciais como desempenho, funcionalidade, estética, qualidade e custo (JOHANSSON, 2002; WEENEN, 1995). $\mathrm{Na}$ última década, diversos métodos e ferramentas de ecodesign (qualquer meio sistemático para lidar com as questões ambientais durante o processo de desenvolvimento de produtos) foram desenvolvidos (BAUMANN; BOONS; BRAGD, 2002; BYGGETH; HOCHSCHORNER, 2006). O intenso desenvolvimento de novos métodos e ferramentas de ecodesign em detrimento do estudo e aprimoramento das existentes e a falta de integração do amplo contexto do desenvolvimento de produtos e a sua ligação com a estratégia da empresa e com os processos de competição e cooperação são duas das explicações citadas por Baumann, Boons e Bragd (2002) para a não obtenção das potencialidades do ecodesign, tal como previsto no começo da década de 1990. Assim, apesar da existência de vários métodos e ferramentas de ecodesign, eles não são utilizados de forma sistemática no desenvolvimento de novos produtos. Para que o Ecodesign seja de fato posto em prática, seus métodos e ferramentas precisam ser identificados e sistematizados, para posteriormente poderem ser devidamente selecionados e aplicados ao processo de desenvolvimento de produtos.

Dessa forma, esse trabalho tem como objetivo geral o levantamento e a classificação dos métodos e ferramentas de ecodesign existentes e aplicados no mundo atualmente, de modo a auxiliar as empresas na seleção e aplicação dos métodos e ferramentas de ecodesign no processo de desenvolvimento de produtos. Esse trabalho faz parte de um projeto mais amplo que visa o desenvolvimento de um Modelo de Maturidade em Ecodesign, que tem como objetivo guiar as empresas na efetiva implementação das práticas de Ecodesign seguindo uma abordagem evolutiva para a aplicação do conceito. O Modelo de Maturidade em Ecodesign é composto por três elementos principais: corpo de conhecimentos de práticas de ecodesign (métodos e ferramentas, práticas de gestão e diretrizes de ecodesign), níveis de maturidade e método de aplicação. Nesse artigo, será apresentada parte do corpo de conhecimento de práticas de ecodesign: os métodos e ferramentas de Ecodesign.

A próxima seção apresenta a metodologia de revisão bibliográfica sistemática utilizada para o levantamento do estado da arte dos métodos e ferramentas de ecodesign. A seção 3 apresenta o planejamento e realização da revisão sistemática e a seção 4 apresenta as conclusões obtidas ao final de revisão. Finalmente, na seção 5, são apresentadas as referências bibliográficas. 


\section{METODOLOGIA}

O principal procedimento técnico utilizado para a realização da pesquisa, identificação e classificação dos métodos e ferramentas de ecodesign é a Revisão Bibliográfica Sistemática, uma metodologia de pesquisa específica, desenvolvida formalmente para levantamento e avaliação de evidências pertencentes a um determinado foco de pesquisa. $\mathrm{O}$ processo de condução da pesquisa em uma revisão sistemática segue uma seqüência bem definida de passos metodológicos, de acordo com um protocolo desenvolvido previamente (BRERETON, KITCHENHAM; BUDGEN, 2007). O modelo de revisão sistemática que será seguido é apresentado pelos autores (BIOLCHINI et al, 2005) e é composto por três fases: (1) planejamento, (2) execução e (3) análise dos resultados. $\mathrm{Na}$ fase de planejamento são definidos os objetivos da revisão e seu protocolo. A fase de execução envolve identificação inicial de estudos, seleção e avaliação de acordo com os critérios de inclusão e exclusão definidos no protocolo de revisão. Uma vez que os estudos tenham sido selecionados, na fase de análise dos resultados são extraídos os dados dos estudos. Os resultados da revisão bibliográfica sistemática devem ser armazenados durante a condução de todas as fases. As ferramentas utilizadas para auxiliar a realização da revisão bibliográfica sistemática foram o uma planilha eletrônica, para o cadastro dos estudos, e um banco de dados, para o cadastro e classificação dos métodos e ferramentas de ecodesign.

\section{REVISÃO BIBLIOGRÁFICA SISTEMÁTICA: MÉTODOS E FERRAMENTAS DE ECODESIGN}

Essa seção é composta pela descrição do modo pelo qual a revisão bibliográfica sistemática foi realizada, seguindo as fases de Planejamento, Execução e Análise dos Resultados.

\subsection{Planejamento}

Essa seção apresenta os passos empreendidos para a elaboração do protocolo de revisão desenvolvido: formulação do problema, coleta de dados, avaliação dos dados, análise e interpretação dos dados e conclusão e apresentação.

\section{Formulação do problema}

O foco de interesse da revisão sistemática realizada, isto é, o objetivo de pesquisa da revisão foi o levantamento e classificação do estado da arte dos métodos e ferramentas do ecodesign existentes e aplicados atualmente. Dessa forma, a pergunta a ser respondida pela revisão sistemática foi: "Quais são os métodos e ferramentas de ecodesign existentes e como eles podem ser classificados?". O alvo da revisão sistemática, de acordo com o seu contexto, pode ser explicitado pelo não atendimento das potencialidades da aplicação do ecodesign, como ganhos econômicos e ambientais, devido à existência e ao 
contínuo desenvolvimento de diversos métodos e ferramentas de ecodesign em detrimento do seu estudo, aplicação e aprimoramento ((BAUMANN; BOONS; BRAGD, 2002). Dessa forma, é fundamental que se conheça e sistematize os métodos e ferramentas existentes de forma que eles possam ser aplicados e aprimorados. A área de desenvolvimento de produtos, incluindo os seus desenvolvedores e projetistas, e a área de gestão ambiental serão as principais áreas de atuação beneficiadas pela revisão sistemática. O resultado esperado com esse trabalho é a identificação, caracterização e sistematização dos métodos e ferramentas de ecodesign existentes e aplicados atualmente. A métrica utilizada para medir o efeito dos resultados da revisão sistemática, foi o número de métodos e ferramentas levantados.

\section{Coleta de Dados}

De acordo com a determinação inicial dos objetivos e do foco do estudo na fase anterior, foram identificadas as bases de dados, as palavras-chave e as expressões lógicas de pesquisa a serem utilizados na revisão. Os termos principais, ou palavras-chaves, que compõe a pergunta da pesquisa são: Eco-design; Ecodesign, Design for environment; Design for the environment; Sustainable product development; Sustainable product design, Life-cycle design; Life cycle design; Green product; Green design; Ecodesign strategies; Eco-design strategies; Design for lifecycle; Design for life cycle; Environmentally product design; Sustainable design; Sustainable product development; Life cycle engineering; Life-cycle engineering. As expressões lógicas que combinam as palavras-chaves e seus sinônimos, que foram utilizadas de forma que fosse obtida a maior quantidade de estudos relevantes foram obtidas pela combinação entre os termos e as palavras methods e tools. O processo de seleção das palavras-chaves e expressões lógicas foi realizado de forma iterativa. Inicialmente, tinha-se um conjunto de 21 artigos, dos quais foram extraídas as palavras-chaves iniciais. Durante o desenvolvimento da revisão, novas palavraschave surgiram e foram adicionadas ao conjunto inicial, resultando na realização novas buscas nas bases de dados com as novas palavras-chave incorporadas. A amostra de população observada durante a intervenção corresponde a bases de dados internacionais, em língua inglesa. O critério utilizado para avaliação das fontes de dados foi a sua abrangência internacional na área de pesquisa. As bases de dados pesquisadas durante a revisão sistemática foram Scirus; Compendex; ISI Web of Science; Research Communications for Scientists and Engineers; Scholar Google Emerald; Find Articles; Science Direct e IEEE Explore. Deve-se salientar, entretanto, a impossibilidade de acesso a alguns estudos encontrados, devido ao fato de que essas bases de dados não são assinadas pela Universidade de São Paulo.

\section{Avaliação dos Dados}

A seleção dos estudos para leitura se deu por meio da aplicação de critérios de inclusão/exclusão de estudos. Foram selecionados aqueles que apresentavam o desenvolvimento de métodos/ferramentas, estudos de caso de sua aplicação, aplicações reais em empresas e estudos de revisão. O procedimento para seleção de estudos com a aplicação dos critérios de inclusão e exclusão foi a leitura do resumo. Quando a leitura do resumo não era suficiente para a inclusão ou exclusão de um artigo, foi realizada a leitura integral do artigo. Assim, aqueles que não 
continham o desenvolvimento de métodos/ferramentas, estudos de caso de sua aplicação, aplicações reais em empresas ou estudos de revisão, eram automaticamente excluídos do conjunto de estudos válidos.

\section{Análise e Interpretação dos Dados}

Para padronizar a forma de representação da informação, foram criados formulários para colecionar os dados dos estudos (planilha eletrônica) e dos métodos e ferramentas de ecodesign (banco de dados). Foram aplicados filtros nos campos desse cadastro, de forma que os estudos pudessem ser buscados mais facilmente de acordo com o tipo de informação necessária, como autor ou palavraschave, por exemplo. O formulário de cadastramento dos métodos e ferramentas de ecodesign foi realizado no formato de banco de dados pela facilidade de exportação dos dados em diversos formatos. Os critérios para classificação dos métodos e ferramentas do ecodesign foram selecionados em função da leitura dos estudos e entendimento das principais funções, características e possibilidades de aplicação dos métodos/ferramentas. Após a seleção inicial dos critérios, ocorreram consultas a especialistas das áreas de meio ambiente e do processo de desenvolvimento de produtos para avaliação da seleção dos critérios mais adequados. Nessa ocasião, foram sugeridos novos critérios que foram adicionados ao formulário de classificação dos métodos/ferramentas. Os critérios utilizados para a classificação dos métodos e ferramentas de ecodesign adotados nesse estudo são:

- $\quad$ Natureza do objetivo principal do método/ferramenta

- Prescritiva: apresentam sugestões genéricas (oriundas de um conjunto pré-estabelecido de melhores práticas de redução de impactos ambientais) para a melhoria do desempenho ambiental de produtos considerando impactos ambientais recorrentes a produtos industriais;

- Comparativa: visam comparar o desempenho ambiental de diferentes produtos, de diferentes conceitos ou de diferentes alternativas de projetos para um mesmo produto;

- Analítica: visam identificar potenciais de melhorias no desempenho ambiental de produtos através da determinação de seus impactos ambientais. As categorias de impactos são pré-estabelecidas de acordo com o método/ferramenta.

- Tipo de ferramenta utilizada pelo método/ferramenta

- Checklist: ferramenta utilizada para checar se um determinado parâmetro relacionado ao desempenho ambiental de um produto foi ou não considerado;

- Guideline: ferramenta que oferece diretrizes gerais a serem seguidas durante o desenvolvimento de produtos para a melhoria de seu desempenho ambiental;

- Matriz: ferramenta que contém uma escala pré-definida para a avaliação do desempenho ambiental de produtos através da relação entre dois aspectos relevantes;

- Software: ferramenta computacional utilizada para suportar a aplicação do método ferramenta;

- Sistemas associados: sistema que possa estar associado ao tipo de ferramenta software.

- $\quad$ Natureza dos dados de entrada e saída: os dados de entrada/saída necessários para um determinado método/ferramenta podem ser qualitativos, quantitativos ou ambos. 
numéricos;

○ Qualitativos: possibilitam análises subjetivas e não necessitam de dados numéricos.

- Quantitativos: possibilitam análises objetivas, com fornecimento de dados

- $\quad$ Área de pesquisa em que foi originado

- Ecodesign/Gestão Ambiental: origem na grande área de pesquisa da gestão ambiental;

- Processo de desenvolvimento de produtos: origem na área de pesquisa do processo de desenvolvimento de produtos, com métodos/ferramentas adaptados dessa área para a consideração da questão ambiental;

- $\quad$ Nível atual de desenvolvimento: critério que avalia o nível atual de desenvolvimento do método/ferramenta em função do seu estado atual de aplicação:

- Teórico: existem apenas estudos acadêmicos teóricos;

- Experimental: aplicado em estudos de caso em caráter piloto em âmbito de pesquisa para validação do seu modelo teórico;

- Consolidado: validado e aplicado regularmente no PDP de empresas;

- $\quad$ Nível de detalhamento do método/ferramenta: nível de detalhamento obtido no estudo em questão

- Superficial: apenas informações gerais do método/ferramenta;

- Sucinto: informações específicas do método/ferramentas, mas de maneira sucinta;

- Completo: informações completas do método/ferramenta.

- Aspectos ambientais considerados: questão aberta para inserção dos aspectos ambientais do produto considerados pelo método/ferramenta (por exemplo, consumo de energia, consumo de materiais, substâncias tóxicas, ruído, vibração, etc.);

- $\quad$ Fases do ciclo de vida consideradas: questão aberta para inserção das fases do ciclo de vida do produto (extração da matéria-prima, indústrias de base, manufatura, uso, descarte, reuso, reciclagem, remanufatura e tratamento e disposição final) consideradas pelo método/ferramenta;

- Método de avaliação de impacto ambiental: critério que verifica se o método/ferramenta possui ou não um método de avaliação de impacto ambiental na sua formulação

\section{Conclusão e Apresentação}

Nesse artigo será dada ênfase para a apresentação das análises conjuntas realizadas dos métodos e ferramentas do ecodesign a partir da classificação realizada de acordo com os critérios apresentados com o objetivo de mostrar um panorama geral e o estado da arte dos métodos e ferramentas desenvolvidos e aplicados atualmente.

\subsection{Execução}

O levantamento bibliográfico de estudos nas bases de dados selecionadas obteve um total de 515 estudos, incluindo artigos, teses, dissertações, livros, etc. Aplicando-se os critérios de inclusão/exclusão, foram selecionados 330 estudos que tratavam de métodos e ferramentas do ecodesign. Realizou-se então uma revisão 
dos artigos não selecionados para garantir que artigos relevantes não tivessem sido excluídos. A leitura dos artigos selecionados resultou na identificação e classificação, de acordo com os critérios selecionados, de 105 métodos e ferramentas de ecodesign. O número de métodos identificados não foi igual ao número de estudos obtidos, pois observou-se a citação de um mesmo método/ferramenta em diversos artigos diferentes. Da mesma forma, existiam artigos que citavam mais de um método e ferramenta, etc.

\subsection{Análise dos resultados}

Essa seção apresenta a análise dos resultados obtidos nos cadastros de estudos e de métodos e ferramentas de ecodesign. A classificação dos 105 métodos/ferramentas realizada possibilitou a análise dos dados em função dos critérios de classificação adotados, apresentada a seguir.

\section{Natureza do objetivo principal}

A figura 1 apresenta a natureza do objetivo principal dos métodos/ferramentas cadastrados e classificados. Percebe-se que os métodos/ferramentas de natureza analítica (como o AT\&T's Green Design Tool (POYNER; SIMON, 1995; KASSAHUN, SAMINATHAN; SEKUTOWSKI, 1995) são mais utilizados do que aqueles de natureza prescritiva (EcoStrategy Wheel (BASTANTE-CECA et al, 2006; HEMEL; CRAMER, 2002; TU; FU-LIN, 1999; LAGERSTEDT, 2003)) ou comparativa (Dominance Matrix or Paired Comparison (BYGGETH; HOCHSCHORNER, 2006). É importante notar, entretanto, que um mesmo método/ferramenta pode ter mais de um objetivo principal, o que possibilita a sua classificação como prescritiva e analítica simultaneamente. O Green Design Advisor (SUN et al, 2003; FERRENDIER et al, 2002; WIXOM, 1994; REGNIER; HOFFMAN, 1998), por exemplo, é analítico e prescritivo ao mesmo tempo.

A AT\&T's Green Design Tool (POYNER; SIMON, 1995; KASSAHUN; SAMINATHAN; SEKUTOWSKI, 1995) analisa o design e os processos associados do ponto de vista ambiental, dando ao designer uma visão geral do status ambiental do design do produto. A EcoStrategy Wheel (BASTANTE-CECA et al, 2006; HEMEL; CRAMER, 2002; TU; FU-LIN, 1999; LAGERSTEDT, 2003), por sua vez, contém 33 princípios agrupados em 8 estratégias que abrangem todo o ciclo de vida dos produtos, guiando os desenvolvedores de produto na tomada de decisão quanto às possíveis melhorias ambientais de um produto. $O$ propósito da Dominance Matrix or Paired Comparison (BYGGETH; HOCHSCHORNER, 2006) é estabelecer um ranking de soluções ou critérios, por exemplo, demandas de um produto ou requisitos ambientais que competem entre si, por meio de uma comparação sistemática entre as diferentes alternativas. Cada alternativa individual é comparada qualitativamente com todas as outras alternativas. O software Green Design Advisor (SUN et al, 2003; FERRENDIER et al, 2002) é usado principalmente para a análise das informações e avaliação do produto, além de ser um ferramenta para divulgação de resultados. Oferece direções para melhorias, assim como características de design com o maior potencial de melhoria, mostrando pontos fracos e fortes do design. Existem ainda guidelines de design adicionais, entretanto, eles não geram 
automaticamente alternativas de design. A solução técnica depende da criatividade do designer e é específica a um produto.

\section{Tipo de ferramenta utilizada}

A figura 2 indica que há uma tendência para o uso de matrizes, ferramenta que contém uma escala pré-definida para a avaliação do desempenho ambiental de produtos por meio da relação entre dois aspectos relevantes, nos métodos/ferramentas do ecodesign, como as matrizes MECO (HOCHSCHORNER; FINNVEDEN, 2003; HOCHSCHORNER, 2004) e MET (BYGGETH; HOCHSCHORNER, 2006; UENO et al, 2001) conhecidas com simplyfied life cycle assessment tools. É importante notar que um mesmo método pode utilizar mais do que um tipo de ferramenta durante a sua aplicação. O DfE Matrix (YARWOOD; EAGAN, 1998) e o Eco-Function Matrix (LAGERSTEDT, 2003), por exemplo, além de terem uma matriz na sua estrutura, apresentam ainda checklists que devem ser utilizados para o preenchimento das células da matriz. Exemplos de guidelines são 10 Guidelines for Ecodesign (PRé Consultants), regras práticas que sintetizam o pensamento do ciclo de vida, e Remanufacturing Guidelines (IJOMAH et al, 2007), que apresenta diretrizes que devem ser consideradas para o desenvolvimento de um produto visando a sua remanufatura, além de características dos produtos que podem auxiliar e/ou impedir a remanufatura. A MECO Matrix (HOCHSCHORNER; FINNVEDEN, 2003) realiza uma estimativa do impacto ambiental de cada fase do ciclo de vida (fornecimento de matéria-prima, manufatura, uso, disposição e transporte) é realizada por meio de estimativas das quantidades de materiais (M), energia $(E)$, químicos $(C)$ e outros materiais $(O)$ utilizados na produção e uso do produto. De forma similar, a MET Matrix (BYGGETH; HOCHSCHORNER, 2006) classifica os impactos ambientais nas categorias Ciclo de Materiais (M), Uso de Energia (E) e Emissões Tóxicas (T) e apresenta uma perspectiva do ciclo de vida para a avaliação qualitativa/quantitativa dos impactos ambientais de um produto. A DfE Matrix (YARWOOD; EAGAN, 1998) levanta questões relacionadas aos impactos ambientais do produto que podem não ter sido considerados previamente através de 100 questões que alocam uma grande gama de tópicos ambientais e de design e fornece uma análise semi-quantitativa das alternativas de design do produto. $O$ resultado é uma pontuação relativa do produto, utilizada para comparar o produto que está sendo desenvolvido com um produto existente, ou ainda para comparar alternativas de design para um novo produto de acordo com o seu impacto ambiental potencial. O Eco-Function Matrix, desenvolvido por Lagerstedt (2003), promove um trade-off entre aspectos funcionais e ambientais do produto em desenvolvimento, visando à incorporação sistemática das propriedades funcionais necessárias ao produto ao menor custo ambiental.

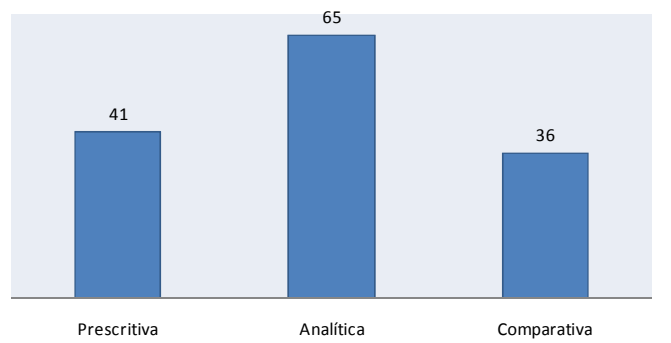

Figura 1 - Natureza do objetivo principal

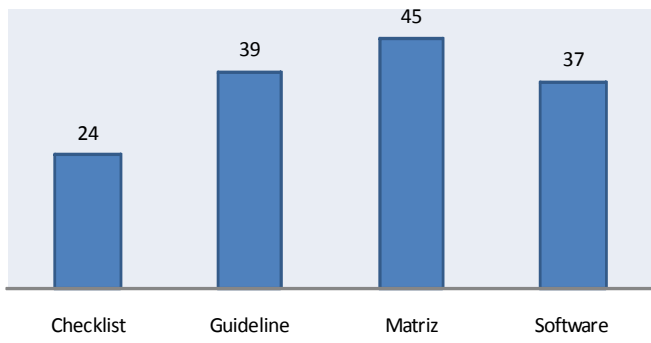

Figura 2 - Tipo de ferramenta utilizada 
A figura 3 mostra um equilíbrio entre a natureza dos dados de entrada e saída necessários para a aplicação de um método/ferramenta. Um mesmo método/ferramenta pode necessitar de dados de entrada e/ou saída qualitativos, quantitativos ou ambos. Geralmente, durante as fases iniciais do processo de desenvolvimento de produtos, devem ser utilizados métodos/ferramentas que tenham dados de entrada preferencialmente qualitativos, devido à falta de informação característica dessas fases (The Ten Golden Rules (LUTTROPP; LAERSTEDT, 2006), por exemplo). Conforme o processo de desenvolvimento vai evoluindo, métodos/ferramentas quantitativos passam a ser adotados para avaliação do desempenho ambiental do produto que está sendo desenvolvido e identificação de pontos fortes para melhoria do seu desempenho. Exemplo de método que exige dados quantitativos é a Life Cycle Assessment (TINGSTROM; KARLSSON, 2006; SAKAO et al, 2002; LEE et al, 2003; GUINÉE; HUPPES; HEIJUNGS, 2001; BREZET; STEVELS; ROMBOUTS, 1999; ANDERSSON et al, 1998).

A ferramenta The Ten Golden Rules (LUTTROPP; LAGERSTEDT, 2006) guia o desenvolvedor de produtos quanto às questões gerais a serem consideradas, consiste de um sumário de diversas linhas guias e manuais utilizados por empresas dos mais diversos setores, contendo recomendações de estratégias ambientais. A Life Cycle Assessment (TINGSTROM; KARLSSON, 2006; SAKAO et al, 2002; LEE et al, 2003; GUINÉE; HUPPES; HEIJUNGS, 2001; BREZET; STEVELS; ROMBOUTS, 1999; ANDERSSON et al, 1998), uma das ferramentas mais famosas e completas para avaliação do impacto ambiental, é uma ferramenta quantitativa de avaliação dos aspectos ambientais e dos impactos potenciais associados a um produto ou serviço, considerando-se os fluxos de matéria e energia durante o ciclo de vida do produto.

\section{Área de pesquisa de origem}

A figura 4 identifica a área de pesquisa de origem dos métodos/ferramentas. A maioria deles tem origem na área do ecodesign, ou da grande área de gestão ambiental. Os métodos/ferramentas com origem no PDP foram, na sua maioria, adaptados de ferramentas bem estabelecidas na área, como o Quality Function Deployment (QFD) e o Failure Mode Effect Analysis (FMEA) ao Quality Function Deployment for Environment (QFDE) (MASUI; SAKAO; INABA, 2001; MASUl et al, 2003; YIM; HERRMANN, 2003; SHIH; LIU, 2005; SAKAO et al, 2002; MASUl et al, 2002; MASUI et al, 2001; FARGNOLI, 2003; BOONKANIT; APIKAJORNSIN, 2005) e Environmental Effect Analysis (EEA/E-FMEA) (LINDAHL, 2001; TINGSTROM; KARLSSON, 2006; LINDAHL, 1999; LINDAHL, 2005), respectivamente. Existem ainda métodos/ferramentas que combinam conceitos tanto da área de gestão ambiental quanto do desenvolvimento de produtos, como o Environmental Design Industrial Template (EDIT) (SPICER; WANG, 1997). O QDFE foi desenvolvido para a incorporação dos aspectos ambientais (requisitos ambientais e especificações de engenharia ambientais) no QFD tradicional para lidar com os requisitos tradicionais da qualidade e com os requisitos ambientais simultaneamente) (MASUI; SAKAO; INABA, 2001; MASUI et al, 2003; YIM; HERRMANN, 2003; SHIH; LIU, 2005; SAKAO et al, 2002; MASUl et al, 2002; MASUl et al, 2001; FARGNOLI, 2003; BOONKANIT; APIKAJORNSIN, 2005). O princípio básico do método Environmental Effect Analysis (LINDAHL, 2001; TINGSTROM; KARLSSON, 2006; LINDAHL, 1999; LINDAHL, 
2005) é listar todas as atividades que podem ter influência ambiental significante, e para cada atividade realizar um julgamento da quantidade e seriedade de cada aspecto, assim como sugerir maneiras para realizar melhorias que podem reduzir os impactos do produto proposto. Após a implementação das ações de melhoria propostas, é realizada uma nova análise. Os impactos ambientais são então reavaliados para checar se as ações realizadas resultaram em melhorias do desempenho do produto, com uma diminuição do seu impacto ambiental total. A idéia geral do Environmental Design Industrial Template (EDIT) (SPICER; WANG, 1997) é realizar uma análise dos efeitos de um produto no fim de vida, gerando uma seqüência de desmontagem para otimizar a geração de lucros e minimizar a geração de impacto. Como resultado, o designer sabe quanto do produto pode ser reusado, remanufaturado, reciclado ou disposto, além do tempo e energia gastos no processo de desmontagem.

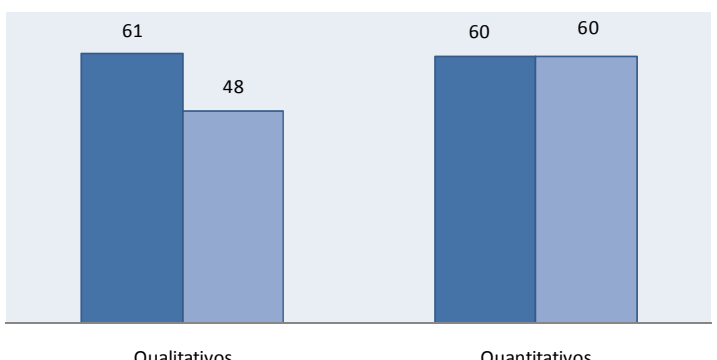

Figura 3 - Natureza dos dados de entrada e saída

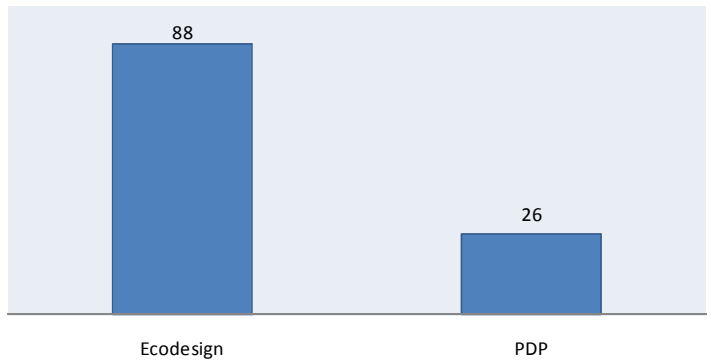

Figura 4 - Área de pesquisa de origem

\section{Nível atual de desenvolvimento}

O nível atual de desenvolvimento dos métodos/ferramentas obtidos e classificados durante a revisão bibliográfica sistemática é apresentado na figura 5 . Percebe-se que, apesar do intenso desenvolvimento teórico e da validação experimental dos métodos/ferramentas, a grande maioria deles ainda não está sendo aplicado regularmente pelas empresas. Esse fato pode ser explicado pela falta de integração dos métodos/ferramentas ao processo de desenvolvimento das empresas. A maioria dos métodos que são aplicados em empresas são aqueles que foram desenvolvidos pela própria empresa, como Philips STRETCH (Strategic Environmental Challenge) (STEVELS, 1999; MEINDERS; STEVELS, 1997; JONES et al, 2001; CRAMER; STEVELS, 1997), Volvo's Black, Gray and White Lists (LUTTROPP; LAGERSTEDT, 2006) e New DFE Targeting and Tracking System HP (NEAL; HEINTZ, 2001).

O Phillips STRETCH (STEVELS, 1999; MEINDERS; STEVELS, 1997; JONES et al, 2001; CRAMER; STEVELS, 1997), realiza um brainstorming ambiental, partindo da identificação das forças prioritárias do negócio (idêntico ao processo de elaboração da estratégia do negócio), passando pela formulação de uma estratégia ambiental e identificando novas oportunidades de negócio. A Volvo's Black, Gray and White Lists (LUTTROPP; LAGERSTEDT, 2006) tem como proposta listar as substâncias químicas que não devem ser usadas, as que têm uso limitado e aquelas que podem ser críticas por um ponto de vista de saúde e ambiental. A base de conhecimentos da New DFE Targeting and Tracking System - HP (NEAL; HEINTZ, 2001) é o coração da ferramenta, e captura os Objetivos de Design Genéricos submetidos pelos Especialistas em Processos de diversas partes de toda a cadeia de valor. A 
avaliação do produto envolve o desenvolvimento de métricas, identificação de questões e a procura para a resolução dessas questões.

\section{Nível de detalhamento}

A figura 6 apresenta o nível de detalhamento dos métodos/ferramentas classificados encontrados no estudo e indica que a maioria dos métodos/ferramentas é apresentada de forma sucinta, como o Eco-roadmap, uma ferramenta gráfica concisa que captura os motivadores ambientais de curto e longo prazo em um documento, contendo a legislação atual relevante ao produto e as necessidades do cliente no escopo da concordância do design com a sustentabilidade e com o meio ambiente (DONNELLY et al, 2006). Essa observação pode ser explicada pela grande quantidade de estudos do tipo artigo obtidos durante a revisão sistemática, em que o espaço para explanações mais detalhadas é limitado. Ainda assim, existem vários estudos em que os métodos/ferramentas são apenas citados e explicados de modo superficial, como aqueles que apresentam revisões sobre métodos e ferramentas de ecodesign. A maioria dos métodos/ferramentas que foram identificados de forma completa são aqueles apresentados em teses, dissertações e livros ou publicações de empresas, como o Eco-Indicator tool, uma ferramenta para calcular o Eco-Indicator (PRE CONSULTANTS, 2000; PRE CONCUSLTANTS et al, 1999; QIAN; YU; ZHANG, 2001). Assim que os dados do produto são informados, o usuário recebe imediatamente o resultado do seu impacto ambiental.

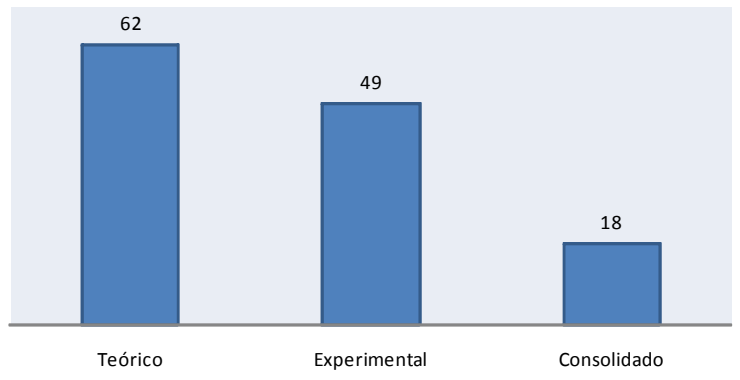

Figura 5 - Nível atual de desenvolvimento

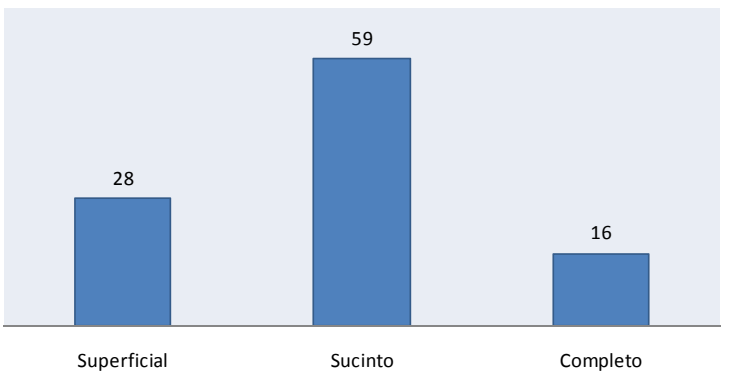

Figura 6 - Nível de detalhamento

\section{Aspectos ambientais considerados}

A figura 7 apresenta os aspectos ambientais considerados pelos métodos/ferramentas classificados. Percebe-se a grande ênfase dada aos aspectos ambientais relacionados ao consumo de materiais $e$ energia nos métodos/ferramentas, como, por exemplo, no grEEEn Method (LICHTENVORT et al, 2003), que possibilita a análise combinada dos perfis ambiental, econômico e de conformidade legal através do fornecimento de informações sobre os impactos ambientais e econômicos durante o ciclo de vida do produto, assim como a legislação específica ao setor. Os cálculos são baseados nos modelos do produto e do processo. Além disso, a elaboração de cenários são parte do método grEEEn para fornecer resultados de avaliações do custo e do impacto ambiental e conformidade legal para alternativas do projeto em vários produtos eletrônicos. A preocupação com os materiais se deve ao interesse em se recuperar a matériaprima do produto após o seu uso, de acordo com as diversas alternativas de fim de vida, como reuso, reciclagem e/ou remanufatura e à existência de legislações 
ambientais, principalmente européias, que regulamentam a responsabilidade do produtor por todo o ciclo de vida do produto. As preocupações com energia são geralmente voltadas à diminuição do consumo durante o uso do produto e à fonte de energia utilizada pelo produto, já que diferentes fontes apresentam diferentes magnitudes de impacto ambiental. Essa preocupação também reflete a busca pela diminuição dos custos do processo de produção do produto, com diminuição da quantidade de materiais e do consumo de energia necessária.

\section{Fases do ciclo de vida}

As fases do ciclo de vida consideradas são apresentadas na figura 8. É importante notar que a maioria dos métodos/ferramentas considera todas as fases do ciclo de vida de um produto, o que pode contribuir para reduções significativas do impacto ambiental ao longo de toda a sua vida e é uma característica do "Life Cycle Thinking". Um exemplo de método que considera todo o ciclo de vida do produto é a Environmentally Responsible Product Assessment Matrix (ERPA) (LEE et al, 2003; HOCHSCHORNER; FINNVEDEN, 2003; HUR et al, 2005). Destaca-se também a grande consideração do fim de vida dos produtos devido a oportunidades de negócios por meio da reciclagem, reuso e/ou remanufatura do produto ou de suas partes, como a End-of-Life Design Advisor (ELDA) (SUN et al, 2003). Para que as estratégias de fim de vida possam ser de fato postas em prática, é necessário que o produto tenha sido desenvolvido para esse fim. A ERPA (LEE et al, 2003; HOCHSCHORNER; FINNVEDEN, 2003; HUR et al, 2005) é utilizada para estimar o potencial de melhorias de um produto de acordo com o seu desempenho ambiental atual e desejado. Cada fase do seu ciclo de vida é avaliada de acordo com cinco critérios (escolha de materiais, uso de energia, resíduos sólidos, efluentes líquidos e emissões gasosas). O impacto ambiental de cada fase do ciclo de vida é estimado através da pontuação de cada critério de 0 (máximo impacto) a 4 (mínimo impacto). Quanto maior for a pontuação de produto, melhor será o seu desempenho ambiental. A ELDA ((SUN et al, 2003) foi desenvolvida para determinar as estratégias de fim de vida do produto durante as fases iniciais do PDP e para fornecer a base técnica para decisões tomadas pelos desenvolvedores de produtos relacionado às tecnologias de tratamento de fim de vida. Através da técnica CART, as características do produto são mapeadas por árvores de decisão, que podem ganhar na predição de estratégias de fim de vida e auxiliar na tomada de decisão. Outra ferramenta específica para a consideração do fim de vida dos produtos com foco para a reciclagem é a Recovery Systems Modeling and Indicator Calculation Leading to End-of-life-conscious Design (ReSICLED) (MATHIEWUX; FROELICH; MOSZKOWICZ, 2006) tem como resultado o cenário de recuperação modelado como uma consistente combinação de processos de recuperação, e converte o fim de vida do produto em material reciclado, energia recuperada e resíduos. A capacidade de recuperação de um produto é quantitativamente avaliada de acordo com três critérios: Indicador de Recuperação em Peso, Indicador de Recuperação Econômica e Indicador de Recuperação de Impacto Ambiental. São considerados ainda, na elaboração de cenários, as incertezas temporais e geográficas. 


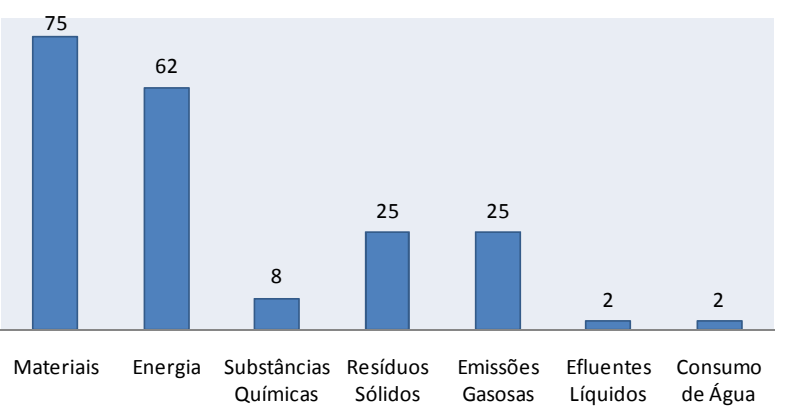

Figura 7 - Aspectos ambientais considerados

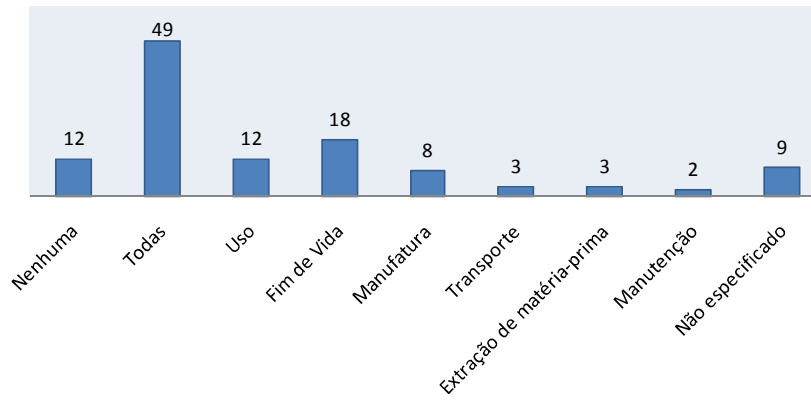

Figura 8 - Fases do ciclo de vida

\section{Método de avaliação de impacto ambiental}

Finalmente, concluiu-se que $83 \%$ dos métodos/ferramentas não possuem um método de avaliação de impacto ambiental. Tal fato faz com que esses métodos/ferramentas sejam mais subjetivos, mais fáceis de aplicar nas fases iniciais do processo de desenvolvimento, onde a quantidade e qualidade das informações ainda são baixas e o grau de incerteza é alto, e sofram maior influência dos conhecimentos e interesses dos usuários, não avaliando sistematicamente o desempenho ambiental dos produtos que estão sendo desenvolvidos. Como exemplo de um método que possui avaliação de impacto ambiental, temos o Environmental Efficiency Potential Assessment method (E2-PA) (NAGATA et al, 2001), uma ferramenta para avaliação do desempenho ambiental de um produto de acordo com os seus impactos ambientais potenciais. A intensidade total corresponde ao desempenho ambiental total do produto como a razão entre a sua utilidade e o balanço total dos recursos envolvidos ao produto por todo o seu ciclo de vida.

\section{CONSIDERAÇÕES FINAIS}

Esse trabalho possibilitou o mapeamento do estado da arte e a classificação dos métodos e ferramentas de ecodesign existentes e aplicados atualmente por meio da realização de uma revisão bibliográfica sistemática, em que foram analisados mais de 500 estudos e sistematizados mais de 100 métodos e ferramentas e ecodesign. Neste artigo, foram citados, em caráter exemplificativo, 25 métodos e ferramentas de ecodesign com diferentes características, objetivos e resultados dos 105 obtidos.

As principais conclusões obtidas como resultado da análise do cadastro dos métodos e ferramentas são: os métodos/ferramentas de natureza analítica são mais utilizados do que aqueles de natureza prescritiva e comparativa; há uma tendência para o uso de matrizes, ferramenta que contém uma escala pré-definida para a avaliação do desempenho ambiental de produtos por meio da relação entre dois aspectos relevantes, nos métodos/ferramentas do ecodesign; há um equilíbrio entre a natureza dos dados de entrada e saída necessários para a aplicação de um método/ferramenta; a origem dos métodos/ferramentas é principalmente na área do ecodesign ou da grande área de gestão ambiental - aqueles com origem no PDP foram, na sua maioria, adaptados de ferramentas tradicionais da área; apesar do intenso desenvolvimento teórico e da validação experimental dos métodos/ferramentas, ainda não há a aplicação regular pelas empresas; a maioria 
dos métodos/ferramentas é apresentada nos estudos de forma sucinta; percebe-se a grande preocupação com os aspectos ambientais relacionados a materiais $\mathrm{e}$ energia; a maioria dos métodos/ferramentas considera todas as fases do ciclo de vida de um produto; $83 \%$ dos métodos/ferramentas não possuem um método de avaliação de impacto ambiental.

O resultado deste trabalho pode contribuir para a seleção dos métodos mais adequados para cada empresa, de acordo com o seu direcionamento estratégico e com os produtos que são desenvolvidos, utilizando-se os critérios de classificação como filtros. Pesquisas futuras podem ser realizadas no sentido de agrupar os métodos e ferramentas com objetivos similares para a criação de métodos únicos para cada necessidade e testar a hipótese acima apresentada.

\section{REFERÊNCIAS}

ANDERSSON, K.; EIDE, M. H.; LUNDQVIST, U.; MATTSSON, B. The feasibility of including sustainability in LCA for product development. Journal of Cleaner Production, v. 6, p. 289-298, set 1998.

BAUMANN, H.; BOONS, F.; BRAGD, A. Mapping the green product development field: engineering, policy and business perspectives. Journal of Cleaner Production, v. 10, p. 409-425, 2002.

BIOLCHINI, J.; MIAN, P. G.; NATALI, A. C. C.; TRAVASSOS, G. H. Systematic Review in Software Engineering. Universidade Federal do Rio de Janeiro. Rio de Janeiro: p. 1-31, 2005.

BOONKANIT, P.; APIKAJORNSIN, A. The Methodology for Selecting Product at Conceptual Design. Fourth International Symposium on Environmentally Conscious Design and Inverse Manufacturing. Tokyo: [s.n.] p. 242-243, 2005.

BRERETON, P.; KITCHENHAM, B. A.; BUDGEN, D. Lessons from applying the systematic literature review process within the software engineering domain. The Journal of Systems and Software, v. 80, p. 571-583, 2007.

BREZET, H.; STEVELS, A.; ROMBOUTS, J. LCA for ecodesign: the Dutch experience. EcoDesign apos; 99: First International Symposium On Environmentally Conscious Design and Inverse Manufacturing. [S.I.]: [s.n.] p. 36-40, 1999.

BYGGETH, S.; HOCHSCHORNER, E. Handling trade-offs in Ecodesign tools for sustainable product development and procurement. Journal of Cleaner Production, v. 14, p. 1420-1430, 2006.

COMMISSION OF THE EUROPEAN COMMUNITIES. Green Paper on Integrated Product Policy. Brussels: 2001.

CRAMER, J. M.; STEVELS, A. L. N. Strategic environmental product planning within Philips sound \& vision. Environmental Quality Management, v. 7, p. 91-102, 1997. 
DONNELLY, K.; BECKETT-FURNELL, Z.; TRAEGER S.; OKRASINSKI, T.; HOLMAN, S. Eco-design implemented through a product-based environmental management system. Journal of Cleaner Production, v. 14, p. 1357-1367, 2006.

FARGNOLI, M. The Assessment of the Environmental Sustainability. 3rd International Symposium on Environmentally Conscious Design and Inverse Manufacturing. Tokyo: [s.n.] p. 362- 368, 2003.

FERRENDIER, S.; MATHIEUX, F.; REBITZER, G.; SIMON, M.; FROELICH, D. Ecodesign Guide: Environmentally improved product design case studies of the european electrical and electronic industry. ECOLIFE Thematic Network. [S.I.] p. 133, 2002.

GUINÉE, J. B.; HUPPES, G.; HEIJUNGS, R. Developing an LCA guide for decision support. Environment Management and Health, v. 12, p. 301-311, 2001.

HEMEL, C. V.; CRAMER, J. Barriers and stimuli for ecodesign in SMEs. Journal of Cleaner Production, p. 439-453, 2002.

HOCHSCHORNER, E. Assessment of tools for Environmentally Preferable Procurement with a Life Cycle Perspective. Royal Institute of Technology. [S.I.] 2004.

HOCHSCHORNER, E.; FINNVEDEN, G. Evaluation of Two Simplified Life Cycle Assessment Methods. International Journal of Life Cycle Assessment, Landsberg, p. 119-128, 2003.

HUR, T.; LEE, J.; RYU, J.; KWON, E. Simplified LCA and matrix methods in identifying the environmental aspects of a product system. Journal of Environmental Management , v. 75, p. 229-237, 2005.

IJOMAH, W. L.; MCMAHON, C. A.; HAMMOND, G. P.; NEWMAN S. T. Development of design for remanufacturing guidelines to support sustainable manufacturing. Robotics and Computer-Integrated Manufacturing, p. 1-8, 2007.

JOHANSSON, G. Sucess Factors for Integration of ecodesign in product development: a review of state of the art. Environmental Management and Health, v. 13, p. 98-107, 2002.

JONES, E.; MANN, D.; HARRISON, D.; STANTON N. An Eco-Innovation Case Study of Domestic Dishwashing through the application of TRIZ tools. Creativity and Innovation Management, v. 10, p. 3-14, mar 2001.

KASSAHUN, B.; SAMINATHAN, M.; SEKUTOWSKI, J. C. Green design tool. Proceedings of the 1995 IEEE International Symposium on Electronics and the Environment. Orlando: [s.n.] p. 118-125, 1995.

LAGERSTEDT, J. Functional and environmental factors in early phases of product development - Eco functional matrix. KTH, Maskinkonstruktion. [S.I.] 2003. 
LEE, J.; KIM, I.; KWON, E.; HUR, T. Comparison of Simplified LCA and Matrix Methods in Identifying the Environmental Aspects of Products. 3rd International Symposium on Environmentally Conscious Design and Inverse Manufacturing. [S.I.]: [s.n.] p. 682-686, 2003.

LICHTENVORT, K.; ALONSO, J. C.; JOHANSSON, G.; BARRUETABENA, L. Applying the grEEEn Method: Initial Results from an Ecodesign Case Study. 3rd International Symposium on Environmentally Conscious Design and Inverse Manufacturing. [S.I.]: [s.n.] p. 636-643, 2003.

LINDAHL, M. E-FMEA - A new Promising Tool for Efficient Design for Environment. First International Symposium On Environmentally Conscious Design and Inverse Manufacturing. Tokyo: [s.n.] p. 734-739, 1999.

LINDAHL, M. Environmental Effect Analysis - How Does The Method Stand in Relation to Lessons Learned from the Use of Other Design for Environment Methods. Second International Symposium on Environmentally Conscious Design and Inverse Manufacturing. Tokyo: [s.n.] p. 864-869, 2001.

LINDAHL, M. Engineering Designers' Requirements on Design for Environment Methods and Tools. Royal Institute of Technology - Department of Machine Design. [S.I.] 2005.

LUTTROPP, C.; LAGERSTEDT, J. EcoDesign and The Ten Golden Rules: generic advice for merging environmental aspects into product development. Journal of Cleaner Production, p. 1-13, 2006.

MASUI, K.; SAKAO, T.; KOBAYASHI, M.; AIZAWA, S.; INABA, A. Quality function deployment for environment QFDE (2ndreport) - verifying the applicability by two case studies. Second International Symposium on Environmentally Conscious Design and Inverse Manufacturing. Tokyo: [s.n.] p. 858-863, 2001.

MASUI, K.; SAKAO, T.; KOBAYASHI, M.; INABA, A. Quality Function Deployment for Environment (QFDE) to Spread DFE on the whole Company. Care Innovation. Vienna: [s.n.] p. 1-6, 2002.

MASUI, K.; SAKAO, T.; KOBAYASHI, M.; INABA, A. Applying Quality Function Deployment to environmentally conscious design. International Journal of Quality \& Reliability Management, v. 20, p. 90-106, 2003. ISSN 1.

MASUI, K.; SAKAO, T.; INABA, A. Quality function deployment for environment QFDE (1st report)- a methodology in early stage of DfE. Proceedings EcoDesign 2001: Second International Symposium on Environmentally Conscious Design and Inverse Manufacturing. Tokyo: [s.n.] p. 852-857, 2001.

MATHIEUX, F.; FROELICH, D.; MOSZKOWICZ, P. ReSICLED: a new recoveryconscious design method for complex products based on a multicriteria assessment of the recoverability. Journal of Cleaner Production, v. 16, p. 1-22, 2006. 
MEINDERS, H. C.; STEVELS, A. L. N. ISO 14001, an oportunity for EcoDesign. International Symposium on Electronics and the Environment. [S.I.]: [s.n.] p. 141-146, 1997.

NAGATA, K.; NOHTOMI, M.; AIZAWA, M.; ASAOKA, K.; USAMI, C. The Development of The Environmental Efficiency Potential Assessment Method. 2nd International Symposium on Environmentally Conscious Design and Inverse Manufacturing. [S.I.]: [s.n.] p. 820-825, 2001.

NEAL, T. L.; HEINTZ, M. A system for integrating design for environment (DFE) criteria into the new product introduction process. International Symposium on Electronics and the Environment. [S.I.]: [s.n.] p. 151-155, 2001.

NIELSEN, P. H.; WENZEL, H. Integration of environmental aspects in product development: a stepwise procedure based on quantitative life cycle assessment. Journal of Cleaner Production, v. 10, p. 247-257, 2002.

POYNER, J. R.; SIMON, M. Integration of DfE tools with product development. CONCEPT - Clean Electronics Products and Technology. Edinburgh: IEE. p. 54-59, 1995.

PRE CONSULTANTS. Eco-indicator 99, Manual for Designers. Pre Consultants. [S.I.], p. 49, 2000.

PRE CONSULTANTS. Ecodesign Tools: tips and tools for environmentally friendly product design. Disponivel em: <http://www.pre.nl/ecodesign/ecodesign.htm>. Acesso em: 15 Fevereiro 2007.

PRE CONSULTANTS. ECO-it Manual. Pre Consultants. [S.I.], p. 32.

QIAN, X.; YU, Y.; ZHANG, H. C. A semi-quantitative methodology of environmentally conscious design for electromechanical products. International Symposium on Electronics and the Environment. [S.I.]: [s.n.] p. 156-160, 2001.

RAM, B.; STEVELS, A.; GRIESE, H.; MIDDENDORF, A.; MULLER, J.; NISSEN, N. $\mathrm{F}$.; REICH, H. Environmental performance of mobile products. International Symposium on Electronics and the Environment. Danvers: [s.n.] p. 140-145, 1999.

REGNIER, E.; HOFFMAN, W. F. Uncertainty model for product environmental performance scoring. Proceedings of the 1998 IEEE International Symposium on Electronics and the Environment. [S.I.]: [s.n.] p. 207-212, 1998.

SAKAO, T.; MASUI, K.; KOBAYASHI, M.; INABA, A. QFDE (Quality Function Deployment for Environment) and LCA: an Effective Combination of Tools for DFE. Care Innovation. Vienna: [s.n.]. 2002.

SHIH, L. H.; LIU, B. S. Evaluating Eco-Design Projects with 3D-QFDE Method and Life Cycle Cost Estimation. Fourth International Symposium on Environmentally Conscious Design and Inverse Manufacturing. Tokyo: [s.n.] p. 722 - 723, 2005. 
SPICER, A.; WANG, M. H. Environmental Design Industrial Template (EDIT): a software tool for analysis of product retirement. Journal of Cleaner Production, v. 5, p. 193-198, 1997. ISSN 3.

STEVELS, A. Integration of EcoDesign into business, a new challenge. First International Symposium On Environmentally Conscious Design and Inverse Manufacturing. Tokyo: [s.n.] p. 27-32, 1999.

SUN, J.; HAN, B.; EKWARO-OSIRE, S.; ZHANG H. C. Design for Environment: Methodologies, Tools and Implementation. Journal of Integrated Design and Process Science, v. 7, p. 59-75, mar. 2003. ISSN 1.

TINGSTROM, J.; KARLSSON, R. The relationship between environmental analyses and the dialogue process in product development. Journal of Cleaner Production, $\mathrm{v}$. 14, p. 1409-1419, 2006.

TU, J. C.; FU-LIN, H. The Ecodesign Strategy on Product Research and Development from the Life-Cycle Design. First International Symposium On Environmentally Conscious Design and Inverse Manufacturing. Tokyo: [s.n.] p. 351356, 1999.

UENO, K.; TAKAHASHI, T.; OYAMA, T.; SHIMAMURA, K.; MATSUMOTO, T.; HASEBE, Y. Efforts to improve the eco-efficiency for products of Mitsubishi Electric Corporation-factor $\mathrm{x}$ by using MET indicators. Proceedings EcoDesign 2001: Second International Symposium on Environmentally Conscious Design and Inverse Manufacturing. [S.I.]: [s.n.] p. 836-841, 2001.

WEENEN, J. C. V. Towards sustainable product development. Journal of Cleaner Production, v. 3, p. 95-100, 1995.

WIXOM, M. R. The NCMS Green Design Advisor, a CAE tool for environmentally conscious design. International Symposium on Electronics and the Environment. [S.I.]: [s.n.] p. 179-182, 1994.

YARWOOD, J. M.; EAGAN, P. D. Design for the Environment - Toolkit. Minnesota Office of Environmental Assistance. Minnesota: p. 72. 1998.

YIM, H.; HERRMANN, C. Eco-Voice of Consumer (VOC) on QFD. Proceedings of EcoDesign2003: Third International Sympsium on Environmentally Conscious Design and Inverse Manufacturing. Tokyo: [s.n.] p. 618-625, 2003. 